\section{Sciensage}

Journal of Advanced Scientific Research

Available online through https: / /sciensage.info
ISSN: 0976-9595

Review Article

DOI: $10.55218 /$ JASR. 202213104

\title{
CURRENT STRATEGIES AND RECENT ADVANCES IN NANOSTRUCTURED DELIVERY SYSTEMS FOR MANAGING LEISHMANIASIS-A REVIEW
}

\author{
Rahul Chaurasia*, Neelam Jain \\ Faculty of Pharmacy, Oriental University, Sanwer Road, Jakhya opposite Revati Range Gate No. 1, Indore, Madhya Pradesh, India \\ *Corresponding author: rahul.chaurasia15@gmai.com
}

\begin{abstract}
Neglected tropical diseases (NTDs) comprise of a group of $17^{\text {th }}$ infectious conditions endemic in many developing countries. Among these diseases, deadly, prevalent parasitic infection such as leishmaniasis, presents a significant global burden which is responsible for high morbidity/mortality rate especially in low-and middle-income/developed countries transmitted by phlebotomines sandflies. As per the WHO report, a total of 0.7-1.0 million new leishmaniasis cases, which are spread by 23 leishmania species in more than 98 countries are estimated with an alarming 26,000-65,000 death toll every year. The disease can be characterized by at least four syndromes: cutaneous leishmaniasis (CL), muco-cutaneous leishmaniasis (MCL), visceral leishmaniasis (VL) also known as kala-azar in the Indian sub-continent or black fever, which is the most firm form of the disease being lethal if untreated and post-kala-azar dermal leishmaniasis (PKDL). The lack of cheap, portable, easy to use diagnostic tools exhibiting high efficiency and specificity impede the early diagnosis of the disease. Its intracellular nature and disseminated locations of parasite, limited number of chemotherapeutic agents, increasing incidences of resistance to first line drugs, along with the cost, toxicities, low patient compliance and require long-term regimen and usually hospitalization, pose a great challenge to formulation scientists that have necessitated effective management of leishmaniasis infection by modulating the delivery of existing drugs. Over the past decade, research on development of alternative treatments such as nanotechnology-based drug delivery systems (nanoparticles, nanotubes, nanosuspensions, liposomes, nanovaccines, etc.), use of natural products as well as development of antileishmanial vaccine has been extensively investigated due to their unique properties, such as bioavailability, lowered toxicity, targeted drug delivery, high biocomatibilty and biodegradability. To identify all relevant literature, we searched Web of Sciences, Scopus, PubMed, NCBI, Scielo, Google Scholar, and profiled studies published between 1986 and 2020. The aim of this review is to summarize new advances and new strategies used on leishmaniasis therapy addressing alternative and innovative treatment paths such as combination or multi-drug uses, immunomodulation, drug repurposing and the nanotechnology-based drug delivery systems have been used to improve the therapeutic aspects of existing antileishmanial drugs, presenting a critical review and some suggestions for improving drug delivery.
\end{abstract}

Keywords: Leishmaniasis, Nanotechnology, Liposomes, Natural products, Drug repurposing, Vaccine.

\section{INTRODUCTION}

Neglected Tropical Diseases (NTDs) represent a group of $17^{\text {th }}$ diseases caused by viruses, bacteria and parasites. NTDs are responsible for a substantial portion of the global health burden and affect more than a hundred nations, approximately 1.4 billion people worldwide, primarily developing countries and areas where communities live in poor sanitary and hygienic conditions [1]. The health burden caused by NTDs is often accompanied by a financial challenge, as developing effective public health approaches to control the disease costs billions of dollars per year [2].
Furthermore, these diseases can cause long-lasting effects that can prevent infected individuals from earning a living, impacting the already precarious socioeconomic situation of many communities [2-4]. Despite the efforts of the international community to control these diseases, NTDs are still a long way from being completely eradicated. Some of the issues related to this are tied to the fact that NTD endemic areas often have unstable political situations and can be subjected to military unrest [5]. Although leishmaniasis is not a household name like malaria, the diseases caused by infection with leishmania continue to have a major 
impact on much of the world's population. Leishmaniasis, one of the most neglected tropical diseases, is currently affecting around 12 million people worldwide and 350 million people are under the risk of infection in 98 developing countries [6]. Leishmaniasis has recently earned more public attention due to its high infection and morbidity rate. Leishmaniasis occurs due to obligate protozoan parasite of the leishmania species [7]. There are almost 51 species of parasites, out of which 21 are pathogenic and cause leishmaniasis [8]. Some of the species that cause leishmaniasis includes $L$. donovani, $L$. amazonensis and $L$. aethiopica etc. Leishmanial parasites exist in two major forms: round and elongated. The round parasite is small and non-motile, while the elongated parasite can move with the help of flagella [9]. Leishmanial transmission occurs when a sand fly sucks blood from an infected individual (human or animal) [10]. The parasite transformation occurs as it changes from the amastigote stage to the promastigote stage, taking about 4-25 days [11]. The disease results in the development of ulcers and also affects other bodily organs [12]. Leishmaniasis exists in 4 major forms, namely as mucosal leishmaniasis (ML), cutaneous leishmaniasis (CL) and visceral leishmaniasis/kala azar (VL) post kala azar dermal leishmaniasis (PKDL). In ML, the symptoms take more time to appear, approximately $1-5$ years. The symptoms include runny nose, ulcers formation, breathing problems and nose bleeding [13]. In CL, the symptoms appear few weeks after the person is bitten by sand fly [14] and in the most common type, VL symptoms appear in about 2-6 months, and include weakness, weight loss, fever, enlarged spleen, liver enlargement, lesions, and swollen lymph nodes [15]. Among endemic regions of the world, 0.2-0.4 and 0.7-1.2 million cases of VL and CL have been reported, respectively. Approximately 75\% of the global estimated prevalence of CL has been reported among certain countries, for example, in Algeria, Afghanistan, Colombia, Syria, Brazil, Iran, Ethiopia, Costa Rica, North Sudan, and Peru, while more than $90 \%$ of VL cases have been reported in Bangladesh, India, South Sudan, Ethiopia, and Brazil $[16,17]$. The leishmanial parasite has ability to take control of the immune system of the affected individuals, which enables the disease condition to persist for a long time and develop into a chronic infection [18]. Basically, the parasite imbalances the host immunity due to its uncontrolled growth inside the macrophages, leading to the eradication of innate, as well adaptive, immunity of the host.
There are two ways by which leishmanial parasite manipulates the immune system; by one way the parasites hide in long-lived macrophage cells surviving hostile conditions [19]. The other way is that the parasite mediates a cell signaling pathway in macrophages which inhibits T-helper cells' (Th2) cytokine responses, specifically interleukins, IL-5, IL-4, and IL-13, leading to down regulation of the protective immune response [20]. Hence, the parasite has the ability to switch between a pro-inflammatory Th1-type healing response to an anti-inflammatory Th2-type nonhealing response, which prioritizes their survival and growth inside the macrophages [21]. Additionally, the parasite has also the ability to inhibit the intracellular leishmanicidal activity by decreasing the production of reactive oxygen species, nitric oxide, and proinflammatory cytokines leading for their better growth and survival by reduced proliferation of CD4+ and $\mathrm{CD} 8+\mathrm{T}$ cells, which eventually leads to an enhanced Th2 response [22, 23]. Furthermore, several co-inhibitory molecules, such as CTLA-4, PD-L1, CD200, and Tim-3 have shifted the balance of the immune system towards the non-healing Th2 response [21]. The lack of knowledge regarding the Th1 to Th2 cell shift in the host immunological response is due to the unidentified host or parasitic factors that contribute to the severe pathology of leishmaniasis. Due to the lack of demarcated entities for protective immunity of the host, the generation of vaccines for the parasite has been a difficult task for researchers. Several leishmania vaccine candidates have been developed and evaluated in native and recombinant form, like gp63, gp46, TSA, PSA2, LACK, LmsT1, Leish111f, and m2, to kill parasites. However, none of them have shown any outcomes towards prophylaxis [24, 25]. Hence, the lack of prophylactic measures has been a concern in the elimination of this NTD. Although the control measures for the elimination of leishmaniasis are limited, yet two strategies have been applied, such as classical therapeutics interventions and vector management through insecticides for the control of the leishmania parasite in disease-endemic regions [26]. The currently available therapeutic interventions are not effective antileishmanial drugs, besides their enhanced number of cases with relapse and repercussions have made the current situation critical for the elimination of leishmaniasis [27, 28]. Thus, the search for safer, more efficient, innovative, cost-effective therapies is urgently needed for treating leishmaniasis. 


\subsection{History and biology of leishmaniasis}

Depending on the disease symptoms, leishmaniasis diagnosis typically falls into one of four major categories: visceral (VL), mucocutaneous (MC), post kala azar dermal (PKDL), or cutaneous leishmaniasis (CL). The earliest Old World records describing lesions with CL character go back to the seventh century BCE. Detailed reports from Arab physicians in the $10^{\text {th }}$ century describe $C L$ in various regions of what is today called the Middle East [29]. Old World VL, or kala azar, characterized by an enlarged spleen, was first recognized in India in 1824. However, the symptoms were confused with those of malaria and attempts were then made to treat the patients with quinine [30]. Clear recognition of VL as a distinct disease was achieved in 1900 after William Leishman and Charles Donovan independently identified leishmania donovani parasites in the spleens of kala azar patients [31]. At about the same time leishmania parasites were also observed in samples obtained from CL lesions. In 1908, Nicolle isolated the parasite from a cutaneous lesion and established the similarity between cutaneous and visceral forms of the disease with regard to the causative agent [32]. The majority of CL cases in the Old World are caused by two leishmania species: L. major and L. tropica. In the New World, CL and MCL cause disfiguring conditions and these have been depicted on sculptures dating back to the fifth century. References to leishmaniasis are also found in the writings of Spanish missionaries from the $16^{\text {th }}$ century [33]. In 1911, Gaspar Vianna discovered that leishmaniasis in South America was caused by a different leishmania species from that in the Old World, and coined a new name, L. brazilienses, for this species [34]. The species name was later corrected to $L$. braziliensis [35]. In the 1960s, additional leishmania species causing CL in Latin America were recognized such as L. mexicana [36]. In 1937, the causative agent of VL in the New World was designated as a distinct species, named L. chagasi [37]. However, this species is indistinguishable from $L$. infantum, the species that causes VL in southern Europe [38]. Leishmania parasites are protozoa belonging to the Kinetoplastida order and Trypanosomatidae family. Over 20 species have been shown to be pathogenic in mammals, with affected hosts including domesticated and sylvatic animals. The parasites are transmitted indirectly between hosts by two different genera of hematophagous sand flies: Phlebotomus and Lutzomyia in the Old and New Worlds, respectively. The life cycle of the leishmania parasite is characterized by two distinct morphologies (Figure 1): the elongated and flagellated promastigote, found in the alimentary tract of the female sand fly vector and the round nonmotile amastigote, present in the bloodstream and tissues of the mammalian host. As an infected sand fly takes a blood meal from a naive host, it regurgitates infective promastigotes at the bite site. The parasites are subsequently taken up by host dendritic cells and macrophages in the dermal layer of the skin. Here, they differentiate into amastigotes and multiply within phagolysosomes (via binary fission) while resisting degradation by lysosomal enzymes. Upon lysis of infected macrophage and dendritic cells, the parasites disseminate via the lymph and circulatory system and go on to infect other macrophages of the reticuloendothelial system. The parasites persist in macrophages present in the spleen, bone marrow, liver and lymph nodes and induce extensive inflammation and increased hematopoiesis [39]. Infected patients serve as parasite reservoirs and can infect naive sandflies when infected macrophages are ingested as part of the sandfly blood meal. After the parasite-infected macrophage is ingested by the sandfly, the amastigotes transform into promastigotes in the insect midgut, multiply and migrate to the proximal end of the gut, where they remain until the next cycle of vector-host infection and transmission [40-42].

\subsection{Detection of leishmaniasis}

The identification of leishmania parasites in culture or in tissues (parasitological diagnosis) is imperative in the diagnosis of leishmaniasis. Traditional methods of low sensitivity used for leishmania detection in clinical samples comprise microscopic techniques (i.e., examination of tissue lesions) [43] and culture techniques. Aspirates from spleen, lymph nodes and bone marrow are typically used and/or liver biopsy is performed. Till 2014 two tests had been applied namely the indirect fluorescence antibody test (BioManguinhos, Fiocruz, Brazil) and the Kalazar Detect rapid test (InBios International, Seattle, Washington, United States) [44, 45]. Up to the present time there exist various rapid diagnostic tests (RDTs) of variable sensitivities for visceral leishmaniasis with a widely applied one being the rK39 [46] which detects antibodies (Abs) against the $L$. chagasi derived recombinant antigen rK39 in VL patients' blood or serum [47]. rK39, enzyme-linked immunosorbent assay (ELISA) and direct agglutination test exhibit good specificities and sensitivities and show comparative results in symptomatic patients whereas the parasite 
detection in asymptomatic patients is still challenging [45]. Isoenzyme electrophoresis is a standard method for the classification of leishmania species, but it is laborious, slow, costly and requires culturing. Various molecular biology techniques like dot, spot, squash blots etc. are characterized by lack of rapidity, specificity and sensitivity [44]. On the other hand, conventional and real-time polymerase chain reaction (PCR) are characterized by high specificity and sensitivity and give reliable quantitative results but are technically complex, require well-equipped laboratories with advanced expensive equipment, and are difficult to interpret. Furthermore, these methods are not usually available in underdeveloped countries [43, 44-48]. In this respect, the development of new portable diagnostic tools allowing the detection of parasites especially in asymptomatic patients is crucial for the early diagnosis and treatment of leishmaniasis [45]. Lately, electrochemical biosensors, where a biological signal is turned into an electrical signal that can be easily monitored and quantified, were developed by immobilizing antigens specifically recognizing antibodies on the sensor surface (e.g., carbon-based electrodes, gold electrodes, screen-printed electrodes). The developed biosensors exhibit advantages compared with traditional methods, like simple construction, portability, rapid response, use of ultra small samples, increased sensitivity and specificity [45, 48]. In addition, combination of electrochemical methods with paper substrate turned out to be a suitable strategy for the development of simple, reasonably priced, disposable and portable diagnostic tools. DNA-based electrochemical sensors have also received a lot of interest because of their ability to detect target DNA sequences, mutated genes and different diseases. Various methods (e.g., adsorption, covalent bonding, etc.) have been utilized to immobilize DNA on the sensor surface. Still, problems like low surface area, insufficient DNA binding, inactive or weak conductive layers, etc., persist and need to be resolved [44].

\subsection{Epidemiology of leishmaniasis}

There are two types of VL that are defined by the causative leishmania species and the parasite reservoir. The zoonotic form, caused by $L$. infantum, occurs in the Mediterranean basin and Central and South America with dogs being the main parasite reservoir $[41,49,50]$. The more common anthroponotic form is caused by $L$. donovani and is predominant in India, Bangladesh, Nepal and East Africa [40, 41]. VL is endemic to rural areas of developing countries and has been reported in approximately 98 countries in the world; $90 \%$ of all cases occur in 6 countries in tropical/subtropical regions: India, Bangladesh, Sudan, South Sudan, Brazil and Ethiopia [40, 41]. Approximately 300000 new cases of VL occur each year leading to an estimated 4000 deaths. India has the highest incidence of the disease with approximately $60 \%$ of all new cases occurring in Bihar state [51, 52]. Outbreaks are common during migration or entry of naive hosts into endemic areas and an increase in the immunosuppressed patient population (such as with HIV) has contributed to the escalation in VL incidence in East Africa [49]. Additionally, an absence of implementation of cost-effective control strategies makes VL a major public health concern [40]. PKDL is prevalent in areas where $L$. donovani is endemic (India and East Africa) and occurs in $50-60 \%$ of Sudanese and 10-20\% of Indian VL patients within 6 months to 2-7 years after initial infection [53-55]. Of these cases, approximately $15-20 \%$ (India) and $8 \%$ (Sudan) of patients do not have a history of VL, indicating the existence of an asymptomatic infection [56]. Few cases of PKDL caused by $L$. infantum or $L$. tropica have been reported [57]. It has been previously shown that the presence of a small population of infected individuals $(0.5 \%)$ may lead to a widespread epidemic of VL infection in India and other regions of Asia; therefore, PDKL patients play a major role in the spread of the disease, and parasite eradication should be a high priority [58]. Approximately 0.7 to 1.2 million cases of $\mathrm{CL}$ occur each year in the Americas, Mediterranean Basin, the Middle East and Central Asia. A large fraction (75\%) of $\mathrm{CL}$ patients resides in the following ten countries: Afghanistan, Algeria, Colombia, Brazil, Iran, Syria, Ethiopia, North Sudan, Costa Rica, and Peru [52]. The disease is caused by $L$. tropica, L. major and L. aethiopica in the Old World (Southern Europe, Middle East, Southwest Asia, and Africa) or by L. mexicana, L. braziliensis and additional leishmania species in the New World (Central and South America) [50]. CL cases caused by L. major and L. tropica (anthroponotic) and by $L$. mexicana are characterized by papulae that typically heal within a few months without medical intervention, whereas $\mathrm{CL}$ caused by $L$. braziliensis is distinguished by lesions that frequently metastasize to mucosal tissues (MCL) and are treated with antileishmanial therapeutics [59]. DCL ( $L$. amazonensis) and MCL are complications of CL that occur primarily in the New World (90\% of cases found in Brazil, Bolivia, and Peru), respectively. An increasing 
number of CL cases have been reported in individuals that have served in the military, international travelers and endemic area migrants $[59,60]$. Travels to Central and South America account for approximately $40 \%$ of CL cases in tourists and workers in the USA [61]. While some cases of leishmaniasis introduced into industrialized nations involve VL, greater than $80 \%$ of these are caused by CL. In fact, CL is one of the most frequent skin disorders in the New World, and accounts for around $60 \%$ of all cases in nonendemic areas [62]. With increasing travel, immigration and military work in endemic areas of this disease, the risk levels and incidence are predicted to increase hence making implementation of precautionary measures crucial in this selected group.

\subsection{Conventional treatment strategies against leishmaniasis and their limitations}

The treatment of leishmanial disease has always been a challenge for researchers. In the early 1950s, sodium stibogluconate and meglumine antimoniate were a few of the first anti-leishmanial drugs, but they had many side effects associated with their intake [63]. With advancement in technology and studies, now, there are many therapeutic drugs available for leishmaniasis treatment, including pentamidine, miltefosine, paromomycin, amphotericin B, and its lipid formulations [64-66]. The typical chemical compounds employed for anti-leishmanial drug development includes antimony sulfide, doxorubicin, quillaja, saponin and phosphatidylserine. Although patients with compromised immune systems, cardiac diseases, and organ transplantation cannot be given drugs like pentavalent antimonial [67], it has been recently found that amphotericin $\mathrm{B}(\mathrm{AmB})$ is the most effective drug for anti-leishmanial activity. AmB was initially used as an antifungal compound consisting of deoxycholate salt [68]. AmB with encapsulation of liposome has been found to be more effective than AmB alone. Liposmal $\mathrm{AmB}$ is less toxic than $\mathrm{AmB}$ alone, although it becomes costly [69]. Miltefosine (Impavido) is the only orally administered anti-visceral leishmanial drug. However, the issue associated with miltefosine is that it cannot be used in pregnant and feeding women because it can harm the developing fetus in the womb [70]. A new drug, Humatin has been developed recently with similar efficiency as AmB but with a limited number of side effects [71].

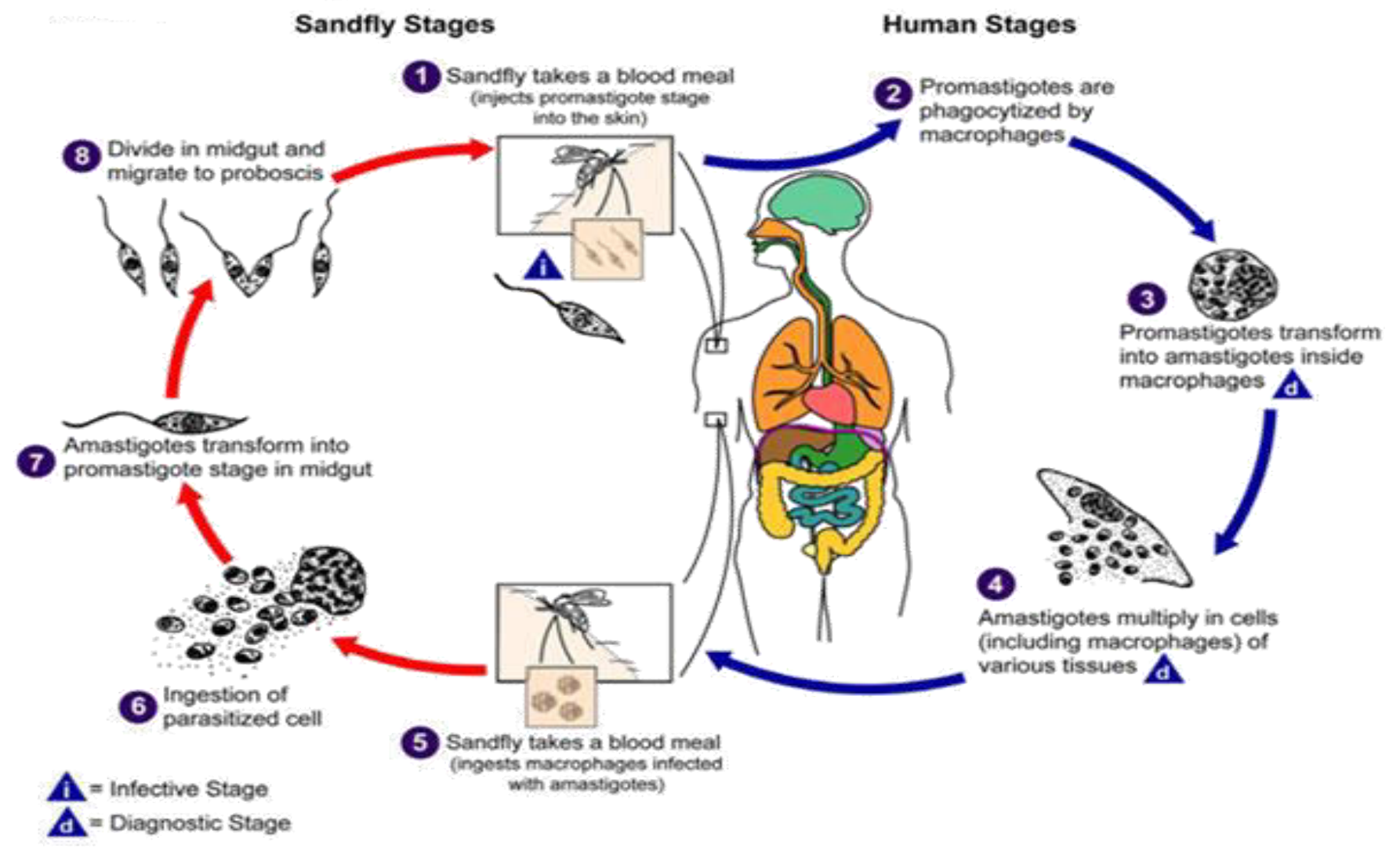

Fig. 1: Life cycle of leishmania parasites (source: Chem Rev. 2014; 114(22): 11305-11347) 
Vaccination is another approach that is employed for leishmanial treatment. The convention vaccine for leishmanial immunization made use of the killed parasite as an antigenic component but its efficacy was low [72]. Later, another approach for fighting the leishmania parasite was introduced, known as a peptide vaccine [73]. The approach is based on the utilization of a minimal pathogenic component to generate long-lasting immunity against the deadly parasite. The choice of epitope is very crucial in peptide vaccine development and, therefore, different in vitro and in silco analysis are conducted to determine the immunogenicity of the peptides [74]. The potential peptides are compared and the best epitope candidates are used for vaccine development by combining multiple epitopes. Peptide vaccine is a promising approach for leishmanial treatment but the challenge is that it is degraded very easily in the body by the immune system [75]. In conclusion, all the available antileishmanial treatments have some limitations or side effects associated with them. Chemotherapeutic drugs are expensive and the parasite has developed resistance against them. Clinical mishandling of medicines in a majority of underdeveloped countries has played a key role in the development of widespread resistance against leishmanial disease [76, 77]. In addition, to date there is no effective vaccine available on the market to prevent leishmaniasis [78]. Thus, it is very important to develop alternative drugs via adopting novel strategies that can effectively control this fatal disease.

\subsection{Novel strategies and current advances on leishmaniasis treatment}

Emergence of resistant strains to conventional drugs [79, 80], high toxicity, co-infections such as HIV/ Leishmania spp., the small therapeutic arsenal available for treatment of the disease and the low investment for the discovery/development of new drugs force researchers and world health agencies to seek new strategies to combat and control this important neglected disease. In this sense, the following section is a brief summary of recent advances and new strategies used to treat leishmaniasis.

\subsection{1. $\mathrm{CO}_{2}$ laser administration and thermotherapy}

Based on the principle to directly destroy the leishmania parasites, the $\mathrm{CO}_{2}$ laser and thermotherapy are a simple way to deliver external heat on infected tissues, causing damage to specific areas with parasitism [81, 82]. The direct application of heat can accelerate the cure of the cutaneous lesions. In OWCL, some studies have demonstrated that thermotherapy showed better results in relation to cure rate compared with intralesional treatment with antimonials with similar or reduced side effects [83, 84]. An improvement was described using a $\mathrm{CO}_{2}$ laser thermotherapy technology, demonstrating in a clinical trial $93.7 \%$ of cure rate compared with combined therapy using intralesional antimonials (78\% of cure rate) [85]. For CL, these strategies have been used with relative success, both for old and new world infections.

\subsubsection{Cryotherapy}

Cryotherapy also known as cryosurgery was firstly evaluated in Saudi Arabia patients infected with leishmania major and obtained $100 \%$ of cure using a $\mathrm{CO}_{2}$ cryomachine [86]. Nowadays, cryotherapy uses liquid nitrogen $\left(\right.$ at $\left.-195^{\circ} \mathrm{C}\right)$, and applied once or twice weekly in leishmania lesions, it can achieve an efficiency rate over 95\% [87]. The mechanism of killing parasites was described by the formation of ice intracellularly causing the disruption of cells leading to localized ischemic necrosis. The secondary side effects were mainly associated with edema and erythema at the site, hyper or hypopigmentation [88].

\subsubsection{Electrotherapy}

Electric field stimulation, a non-drug treatment, has been described as a potential tool to control microbial infection. Previous test using mice infected with Pseudomonas aeruginosa resulted in significantly inhibiting bacteria in lung infections [89]. Authors have shown that exposure to electrical currents could lead to healing the skin lesions and intractable ulcers and the new skin produced has better tensile properties compared with that skin produced naturally [90]. In leishmaniasis, the use of therapeutic electricity applied on infected mice with L. major showed important death of parasites at the lesion sites [91]. Recent advances have demonstrated that electric fields affected leishmania tarentolae promastigote motility, clumping and viability in vitro [92]. However, these studies are in an initial phase requiring more data related to what currents, potentials, numbers of stimulations, and durations are safe but effective for clinical use against CL.

\subsubsection{Nitric oxide derivates}

This is a promise strategy used against some leishmania species from Americas (L. braziliensis and L. panamensis). A S-nitroso- $\mathrm{N}$-acetylpenicillamine cream that generates 
NO was firstly tested for 10 days showing, after 30 days of treatment, the healing of all lesions and the formation of new skin in patients presenting CL. Moreover, a most recent study using a topical nanofiber nitric oxide (NO)-releasing patch administered for $12 \mathrm{~h}$ a day for 20 days showed ineffectiveness, with only $37.1 \%$ of cure rates in CL Colombian patients [93, 94]. The authors suggest that therapeutic failure can be reversed by increasing drug concentration or treatment time.

\subsubsection{Intralesional drug administration}

Thinking of reducing the adverse effects while maintaining efficacy and safety of the form of conventional use (intramuscular or intravenous infusion), this new way to treat $\mathrm{CL}$ using pentavalent antimonials has gained prominence. Since 2013, the use of intralesional antimonial therapy has been recommended by the PAHO guidelines [95] when systemic treatment is not indicated or if local treatment for $\mathrm{CL}$ is required. Intralesional pentavalent antimonials achieved cure rates over $90 \%$ against Asia and Mediterranean species of the parasite (L. major and Leishmania tropica) [96]. For new world leishmania species, open-label phase II clinical trials showed elevated cure rates (87-91.6\%) after 180 days of treatment [97]. The most common side effects observed were bacterial secondary infection, erythema, local itching, and pain during administration, which tend to disappear few days after the end of the treatment [98].

\subsubsection{Multi-drug or combination therapy}

It is well known that untreated symptomatic VL is almost always fatal. Moreover, it is observed a large range on the variability in the effectiveness of antileishmanial drugs associated with the region where the leishmania infection occurred and the host immune status [99]. In this sense, combined therapy has the following objectives: shortening the treatment duration (reducing side effects and improved adherence to the regimen by the patient), controlling the development of parasite resistance, lowering the costs and encouraging a cure, especially in complicated cases of VL [100]. The combined therapy is mainly recommended for patients who had not responded to monotherapy with Sbv. Most of the studies were conducted in India especially in leishmania donovani-infected patients. Using AmB-L at 5 $\mathrm{mg} / \mathrm{kg} /$ day in a single dose followed by different regimens of miltefosine, the authors demonstrated greater efficacy with combinations compared with AmB-L at $5 \mathrm{mg} / \mathrm{kg} /$ day in a single dose (98\%versus
91\%) [101]. In Eastern Africa, some studies have demonstrated that the combination of paromomycin with SSG increased the cure rate response in comparison with SSG asmonotherapy [102]. A recent study in VL/HIV co-infection demonstrated reduced rates of mortality and VL relapse when AmB-L (AmBisome) and miltefosine (Impavido) were combined and administered. Moreover, the authors concluded that combination therapy appeared to be well tolerated, safe, and effective and may be considered as an important option for treatment of VL in HIV coinfected patients [103].

\subsubsection{Immunomodulators}

Leishmania parasites have stated systematic resistance against the immune system manipulating different mechanisms to survive into the host. In this way, treatments with substances that promote the restoration of the immune response against the parasite are an alternative approach to combat the infection. The IFN- $\gamma$ is well recognized as a cytokine capable of inducing macrophages to kill Leishmania parasites. In VL patients, the use of IFN- $\gamma$ as immunotherapy promoted accelerated parasitological control and enhanced the clinical efficacy of conventional Sbv therapy, promoting more than $80 \%$ cure rate. More recently, in a case report on $\mathrm{HIV} / \mathrm{VL}$ co-infection in Italy, the combination treatment using rHuGM-CSF (recombinant human granulocyte macrophage colony stimulating factor) showed to be effective leading to a reduction on the spleen size, disappearance of symptoms, and clinical cure of the patient. Taking into account the use of immunomodulator, the most used strategy in leishmaniasis is therapeutic vaccines. In literature, many studies around the world describe important results obtained using vaccines as immunotherapeutic tool. More than 5000 patients were treated against CL with heat-killed leishmania parasites plus BCG in Venezuela with an incredible $95.7 \%$ of cure rate achieved. In the same way, patients infected with $L$. braziliensis were treated with a therapeutic vaccine composed of parasite derived antigen Fraction 2 (LbbF2) that promoted secretion of key cytokines by $\mathrm{T}$ cells leading to clinical cure of the infected patients. In Brazil, more than 500 patients with CL were treated either with pentavalent antimony, killed Leishmania vaccine plus BCG, BCG, or a combination. The cure rates in therapeutic vaccine or pentavalent antimony chemotherapy were the same, but with fewer adverse effects and shorter recovery time [104]. As observed in these studies, activation of the 
immune system through immunotherapy associated with application of antileishmanial drugs can solve the complicated cases of the disease mainly in patients with drug refractory leishmania infections.

\subsubsection{Drug repurposing}

Drug repurposing is regarded as one of the most important strategies for the rational use of drugs, especially against neglected diseases, called as drug reprofiling or repositioning is a modern strategy to identify and develop new uses for existing drugs [105]. In addition, repositioning has main advantages, including lower risk of drug failure, reduced time frame for development/application of the drug, and reduced costs and can reveal new pathways and targets that may be further explored [106]. Focusing on leishmaniasis drug repurposing, computational approaches are the main strategies that have been applied with relative success among the diseases. These computational techniques involve systematic analysis of any data type such as bioinformatics targeting gene expression, chemical structure and genotype or proteomic data. In this sense, molecular docking is a computational strategy to predict binding sites between the ligands (drugs, for example) and the target (a receptor) [107]. In conventional docking, one receptor/protein target is chosen so that multiple drugs could be tested against that target. In this case, the knowledge about the target/protein or the drug class may favor the choice of a possible ideal drug with a greater chance of success in subsequent trials [106]. Another important tool is called signature matching that is based on the comparison between the characteristic (signature) of a drug or molecule compared with that of another drug or molecule [108]. The signature analyses are derived from some general data such as metabolomic, proteomic, transcriptomic, or chemical structures. These two drugrepositioning tools are the most used strategies against leishmaniasis currently. In a recent study, Bustamante et al. used bioinformatic predictions to detect some repurposing drugs for leishmaniasis treatment. In this study, the authors performed some simulations to identify and predict these drugs with in vitro validations and pharmacokinetic simulations. As strategy, the bioinformatic predictions were used to detect potential homologs between targeted proteins by approved drugs and other proteins of the leishmania spp. parasites. In this study, 33 drugs were identified with potential target prediction with in vitro action (rifabutin and perphenazine) [107]. Metallodrugs have been identified with important antitumor, anti-inflammatory, and antimicrobial actions. Auranofin (Ridaura), a gold (I) triethylphosphine thiosugar drug, has been described as having antileishmanial activity with the ability to inhibit trypanothione reductase (TR). In this sense, a recent study performed a preclinical evaluation of gold (I) triphenylphosphine- and triethylphosphine-based complexes showing their activity against leishmania infantum and $L$. braziliensis intracellular amastigotes. Using bioimaging, the authors observed reduced lesion size and parasite burden in BALB/c mice infected with luciferase expressing $L$. braziliensis or leishmania amazonensis and orally treated with gold (I) complexes. According to the authors, the gold (I) complexes are promising antileishmanial agents, with a potential for therapeutic use [109]. An interesting review discussing drug repurposing has recently been published and describes multi-target drugs active against leishmaniasis. It is shown that the azoles presented growth inhibitory activity against both fungi and leishmania. Some compounds such as posaconazole, fluconazole, and itraconazole act against the same target, lanosterol 14-ademethylase enzyme. Similarly, it is shown that both amphotericin B and miltefosine act on small molecules, proteins, genes and even organelles showing their profile of multi-target agents as known. The authors concluded that some steps towards drug repurposing for multi-target strategy will be the future in the search for leishmanicidal drug candidates [110].

\subsubsection{Promising natural products}

Many people in urban areas rely mainly on standard treatments to improve the symptoms, chiefly the use of medicinal plants. The natural constructs are likely sources of wide chemistry with an extraordinary diversity and convenience in nature. Recently, the Tropical Diseases Program of the World Health Organization (TDR/WHO) with the Drug Discovery Research Program has deliberated a priority for the pharmacological investigation of plants. Widespread studies on movement of natural products next to leishmania during the last years have been accumulated. Recently, the most advances in this field have been outstandingly reviewed and listed plants and natural products derived that showed some levels of antileishmanial activity. Some studies showing the search of new products in microorganism or marine sources, such as a glycoprotein isolated from the sponge Pachymatisma johnstonii, which showed a high activity in vitro against $L$. donovani, $L$. braziliensis and $L$. mexicana 
[111] and aphidicolin a fungal metabolite isolated from Nigrospora sphaerica, which subdued the increase of promastigotes and amastigotes of $L$. donovani. Brazilian, Mexican, Columbian and Peruvian flora extracts showed the antileishmanial activity of plants used by people from common areas of Latin America. The antileishmanial activity of essential oil has been evaluated and facts have been reviewed by Antony and col. [112]. The oil of Croton cajucara, a plant used in folk Brazilian medicine, causes the inhibition of $L$. Amazonensis and increase the nitric oxide production. Nerolidol is a compound present in the essential oils of some plants and inhibits the in vitro growth of $L$. amazonensis, $L$. braziliensis and L. chagasi. Their mechanism of action could be the detachment of earlier steps of ergosterol synthesis. Other advanced studies have evaluated potential compounds isolated from natural source, which displayed antileishmanial activity.

\subsubsection{Nanotechnology: a new horizon for manage- ment of leishmaniasis}

Innovations in interdisciplinary sciences have been moving the translational sciences to the next level for better control of infectious diseases. Nanomedicine (the use of medical applications of nanotechnology for human welfare) is one of the promising fields in this area that has been continuously growing, keeping up hope for highly sensitive diagnostic tools and better drug delivery for various infectious diseases in the near future [113]. As the traditional antileishmanial drugs have low tolerability, long treatment duration, and are difficult to administer, a tremendous upsurge has been observed in the development of novel nano-biopharmaceuticals that can cure leishmaniasis. The field of nanotechnology has played a vital role in revolutionizing the process of delivering drug in the field of medicine. The nanotechnology employs the use of various drug-loaded nanocarrier systems, such as metallic nanoparticles, liposomes, nanoemulsions, nanosphere, solid-lipid nanoparticles, nanocapsules, polymeric nanoparticles and nanostructured lipid carriers and nanostructured layered films for efficient drug delivery to the target sites for the treatment of leishmaniasis. These nanocarrier systems enable targeted delivery, increased bioavailability and reduced toxicity of drugs [114]. Nanocarriers enclose the drugs that provide targeted delivery and also protect the drug from being metabolized [115]. The absorption and distribution profile of nanocarriers greatly depends on the physicochemical properties, i.e., the size, hydro- phobicity, targeting molecule, and their charges. Many processes, like uptake and entry of nanocarriers into cell and their further interaction with immune system are dependent on the size and charge of the nanocarriers. Another property is the hydrophobicity, which controls the absorption and distribution of nanocarriers by effecting the immune cells' interaction, protein interaction, particle clearance and protein charge $[116,117]$. The charge is used in binding plasma protein, protein interactions, membrane damage and in immune cell stimulation [118]. These drug-loaded nanocarriers enter the cell by phagocytosis, a form of endocytosis in which the cell engulfs particles larger than $0.75 \mu \mathrm{m}$ in diameter. Macrophages, neutrophils, and monocytes are capable of phagocytosis and, therefore, sometimes are referred as professional phagocytes [119]. Leishmaniasis is a particularly interesting disease to be treated with drug-loaded nanocarriers since the parasites exclusively infect the highly phagocytic cells known as macrophages. In this way, the macrophages take up the drug-loaded nanocarrier by phagocytosis, where they will directly act on the parasites. This allows the drugs to reach an effective intracellular concentration, along with a reduction in toxicity and dosage of drugs [120, 121]. Furthermore, there are two types of vectoring procedure, active and passive vectoring, which could affect the distribution and uptake. In active vectoring, a specific compound is added or attached to the surface of the nanoparticle, whereas passive vectoring is the inherent capacity of cells when they recognize the foreign particles to organisms. Various nanocarrier systems have been synthesized and used in the controlled drug delivery in treatment of leishmaniasis. Among the traditional nanoparticles, the most preferred are liposomes and polymeric nanoparticles as they are easily and rapidly internalized by macrophages in the liver and spleen [122]. The most commonly employed nanocarriers in curing leishmaniasis are liposomes due to its unique properties. They are able to load and deliver both hydrophobic and hydrophilic drugs by surface functionalization, which is used to improve drug targeting. Additionally, the fate of liposomes and the leishmaniasis parasite is the same. The positivelycharged liposomes are readily taken in by the macrophages. Since the macrophages can recognize sugar molecules, liposomes are surface functionalized with sugar to improve macrophage targeting. However, liposomes face some limitations, as well. They are not stable. They could result in toxicity because the drug can leak from the liposomes into the blood supply [123]. 
Nanoemulsions are one of the best drug delivery systems due to their simple preparation, ability to solubilize hydrophobic drugs, physicochemical stability, and easy scale-up [124]. Polymeric nanoparticles are also a widely used nanoparticle system for the treatment of leishmaniasis. They have the properties to overcome some of the drawbacks of liposomes [125]. They have small size, low toxicity, and are cost effective as they can be used to deliver more than one drug. They have the ability to design biodegradable systems and can be surface functionalized. Among the polymeric nanoparticles, poly lactide-co-glycolide (PLGA) is the most commonly employed as it is biodegradable and biocompatible. One important difference between liposomes and polymeric nanoparticles is their stability. Unlike the unstable nature of liposome, polymeric nanoparticles do not face the limitation of drug leakage into the blood supply [126,127]. There are some advanced nanocarrier systems, such as metallic nanoparticles, dendrimers, and carbon-based nanomaterials. They need to be studied well in order to know their advantages and drawbacks. One important advantage of dendrimers is their ability to load more than one drug due to their branched structure, enhancing drug bioavailability [128]. Along with the advantages of nanocarriers as efficient antileishmanial drugs there are also some challenges to overcome. One of the prominent hurdles is the high cost of these nanoformulations. Hence, their commercialization and high scale production is not economically feasible. On account of their economic feasibility, solid-lipid nanoparticles (SLNs) are better because they are made of triglyceride lipids whose production scale up is less expensive then phospholipids [129].

\subsubsection{Nanovaccines}

Nanovaccines are emerging as a novel approach to the methodology of vaccination, having shown promising results in inducing both humoral and cell-mediated immune responses. These positive results have led to a promising path to more suitable treatment for several diseases, including leishmaniasis [130]. In a 2011 study, recombinant leishmania superoxide dismutase (SODB1) was loaded onto chitosan nanoparticles by the ionotropic gelation method to develop a new nanovaccine that was experimented on BALB/c mice. The results showed that in single and triple doses of SODB1 nanoparticles, IgG2a and $\operatorname{IgG} 2 \mathrm{a} / \operatorname{IgG} 1$ were significantly higher than the other groups $(\mathrm{P}<0.05)$, which shows the efficiency of chitosan nanoparticles in developing a nanovaccine for leishmaniasis [131]. Another study perused the effectiveness of chimeric peptides containing HLA-restricted epitopes from three immunogenic $L$. infantum proteins, in poly (lactic-coglycolic) acid nanoparticles with or without the adjuvant monophosphoryl lipid A (MPLA) or surface modification. The nanovaccine induced dendritic cells maturation and promoted peptide-specific ifn $\gamma$ producing CD8 $+\mathrm{T}$ Cells [132]. Another study used synthetic peptide-based nanovaccines along with MPLA adjuvant co-encapsulated in PLGA nanoparticles. The results demonstrated a strong spleen lymphoproliferative response and high levels of IL-2, IFN- $\gamma$, and TNF $\alpha$ versus low IL-4 and IL-10 secretion [133]. A 2019 study developed a process to prepare lipidic nanoparticles (NPs) loaded with plasmid pVAX1-NH36 for application as a leishmaniasis nanovaccine. The result presented stability N84\% in all of the samples, which could be a promising approach for future studies [134].

\section{CONCLUSION}

There are few drugs for the leishmaniasis treatment and the great toxicity and side effects put in check the international treatment control. Moreover, the emergence of resistant strains to conventional drugs, coinfections such as HIV/Leishmania spp., the small therapeutic arsenal, and the low investment for the discovery/development of new drugs force researchers and world health agencies to seek new strategies to combat and control this important neglected disease. In this context, new strategies with important advances in physical and local therapies including thermotherapy and $\mathrm{CO}_{2}$ laser administration and topical drug therapies using NO compounds and intralesional drug administration have given a better perspective of cure in patients with CL. Moreover, the use of combination therapy or multi-drug therapy and activation of immune system using immunomodulators have helped to solve problems in relation to parasitic resistance and serious cases of HIV/Leishmania spp. infection. Finally, being considered as the future of leishmaniasis treatment, the drug repurposing and the nanotechnology-based drug delivery systems bring the opportunity to use computational tools for the identification of existing drugs which are used in the treatment of the disease with less time, cost, and using nanotechnology that promotes an efficient delivery of different types of drugs to specific tissues and cells infected by the leishmania parasites. Thus, efforts need to be directed for the rational investment in new therapies and treatment 
strategies against the disease, in order to seek therapies with less side effects, lower costs, and better efficacy against these parasites.

\section{Conflict of interest}

None declared

\section{REFERENCES}

1. Buscaglia CA, Kissinger JC, Aguero F. Trends Genet., 2015; 31(10):539-555.

2. Okwor I, Uzonna J. Am. J. Trop. Med. Hyg., 2016; 94(3):489-493.

3. Franco JR, Simarro PP, Diarra A, Jannin JG. Clin. Epidemiol., 2014; 6:257-275.

4. Utzinger J, Becker SL, Knopp S. Swiss Med. Wkly. 2012; 142:w13727.

5. Hotez PJ. PLoS Negl. Trop. Dis., 2017; 11(12): 00005896 .

6. Alvar J, Vélez ID, Bern C, Herrero M, Desjeux P, Cano J. PLoS One. 2012; 7:e35671.

7. Handman E. Cell biology of Leishmania. In Advances in Parasitology; Elsevier: Amsterdam, the Netherlands, 1999; 44:1-39.

8. Vannier-Santos M, Martiny A, Souza WD. Curr. Pharm. Des., 2002; 8: 297-318.

9. Alexander J, Satoskar AR, Russell DG. J. Cell Sci., 1999; 112:2993-3002.

10. Bates PA. Int. J. Parasitol., 2007; 37:1097-1106.

11. Bailey F, Mondragon-Shem K, Hotez P, Ruiz-Postigo JA, Al-Salem W, Acosta-Serrano, A. PLoS Negl. Trop. Dis., 2017; 11:e0005739.

12. Singh K, Garg G, Ali V. Curr. Drug Metab., 2016; 17: 897-919.

13. Blum J, Lockwood DN, Visser L, Harms G, Bailey MS, Caumes E. Int. Health. 2012; 4:153-163.

14. Layegh P, Moghiman T, Ahmadian Hoseini SA. J. Infect. Dev. Ctries., 2013; 7:614-617.

15. Leta S, Dao TH, Mesele F, Alemayehu G. PLoS Negl. Trop. Dis., 2014; 8:e3131.

16. Pigott DM, Bhatt S, Golding N, Duda KA, Battle KE, Brady OJ. Elife. 2014; 3:e02851.

17. Singh OP, Singh B, Chakravarty J, Sundar S. Infect. Dis. Poverty 2016; 5:19.

18. Litt E, Baker MC, Molyneux D. Trends Parasitol., 2012; 28:195-201.

19. Gutierrez V, Seabra AB, Reguera RM, Khandare J, Calderon M. Chem. Soc. Rev., 2016; 45:152-168.

20. Desjeux P. Comp. Immunol. Microbiol. Infect. Dis., 2004; 27:305-318.

21. Gannavaram $S$, Bhattacharya $P$, Ismail N, Kaul A, Singh R, Nakhasi HL. Front. Immunol., 2016; 7:187.
22. Rodrigues V, Cordeiro-da-Silva A, Laforge M, Silvestre R, Estaquier J. Parasites Vectors 2016; 9:118.

23. Tiwari N, Gedda MR, Tiwari VK, Singh SP, Singh RK. Mini Rev. Med. Chem., 2018; 18:26-41.

24. Nagill R, Kaur S. Int. Immunopharmacol., 2011; 11:1464-1488.

25. Singh B, Sundar S. Vaccine. 2012; 30:3834-3842.

26. Gedda MR, Singh OP, Srivastava ON, Sundar S. Therapeutic Leishmaniasis: Recent Advancement and Developments in Nanomedicines. In Nano-technology in Modern Animal Biotechnology; Springer: Berlin/Heidelberg, Germany, 2019; 195-220.

27. Singh N, Mishra BB, Bajpai S, Singh RK, Tiwari VK. Bioorg. Med. Chem., 2014; 22:18-45.

28. Singh OP, Hasker E, Boelaert M, Sundar S. Lancet Infect. Dis., 2016; 16: e304-e309.

29. Manson-Bahr PEC. The Wellcome Trust illustrated history of tropical diseases; Cox, F. E. G., Ed., The Wellcome Trust: London, U.K., 1996.

30. Ross MR. Report on the nature of kala azar; Office of the superintendent of government printing: Calcutta, India, 1899; 1-104.

31. Hoare CA. Trop. Med. Hyg., 1938; 32:66.

32. Nicolle CCR. Hebd. Seances Acad. Sci., 1908; 146:842.

33. Lainson R. The Wellcome Trust illustrated history of tropical diseases; Cox FEG, Ed., The Wellcome Trust: London, U.K., 1996.

34. Vianna G. Bras. Med., 1911; 25:411.

35. Matta AA. Bull. Soc. Pathol. Exot. Ses. Fil. 1916; 9:494.

36. Lainson R, Strangways-Dixon J. Trans. R. Soc. Trop. Med. Hyg., 1964; 58:470.

37. Cunha AM, Chagas E. Nota Previa Hosp., Rio de Janeiro., 1937; 11:3.

38. Kuhls K, Alam MZ, Cupolillo E, Ferreira GE, Mauricio IL, Oddone R. PLoS Neglected Trop. Dis., 2011; 5:e1155.

39. Cotterell SE, Engwerda CR, Kaye PM. Blood. 2000; 95:1642.

40. Chappuis F, Sundar S, Hailu A, Ghalib H, Rijal S, Peeling RW. Nat. Rev. Microbiol., 2007; 5:873.

41. Van Griensven J, Diro E. Infect. Dis. Clin. North Am., 2012; 26:309.

42. Kedzierski L. Hum. Vaccines 2011; 7:1204.

43. Saleem K, Khursheed Z, Hano C, Anjum I, Anjum S. Nanomaterials. 2019; 9:1749.

44. Farshchi F, Saadati A, Hasanzadeh M. Anal. Methods. 2020; 12:4759-4768.

45. Martins BR, Barbosa YO, Andrade CMR, Pereira LQ, Simao GF, de Oliveira CJ. Biosensors. 2020; 10(8):81.

46. Singh OP, Gedda MR, Mudavath SL, Nath O, Sundar SS. Nanomedicine. 2019; 14(14):1911-1927. 
47. WHO Leishmaniasis, medical devices: https:// www.who.int/medical_devices/diagnostics/ selection_in-vitro/selection_in-vitro-meetings/ subid-67-40/en.

48. Mobed A, Mehri P, Hasanzadeh M, Mokhtarzadeh A. J. Mol. Recognit., 1998; 33: e2839.

49. Sundar S, Chakravarty J. Expert Opin. Pharmacother., 2020; 14:53.

50. WHO Expert Committee on the Control of Leishmaniases, Control of the leishmaniases; WHO Tech. Rep. Ser.: Geneva, Switzerland, 2010; 949.

51. Ejazi SA, Ali N. Expert Rev. Anti-Infect. Ther., 2013; 11:79.

52. Alvar J, Velez ID, Bern C, Herrero M, Desjeux P, Cano J. PLoS One. 2012; 7:e35671.

53. Zijlstra EE, Musa AM, Khalil EAG, El Hassan I. Lancet Infect. Dis., 2003; 3:87.

54. Singh S, Sharma U, Mishra J. Int. J. Dermatol., 2011; 50:1099.

55. Ramesh V, Mukherjee A. Int. J. Dermatol., 1995; $34: 85$.

56. Zijlstra EE, el-Hassan AM, Ismael A. Am. J. Trop. Med. Hyg., 1995; 52:299.

57. Sacks DL, Kenney RT, Kreutzer RD, Jaffe CL, Gupta AK Sharma MC. Lancet. 1995; 345:959.

58. Dye C, Wolpert DM. Trans. R. Soc. Trop. Med. Hyg., 1988; 82: 843.

59. Weina PJ, Neafie RC, Wortmann G, Polhemus M, Aronson NE. Clin. Infect. Dis., 2004; 39:1674.

60. Ul Bari AJ. Pakistan Assoc. Dermatol., 2006; 16:156.

61. Herwaldt BL, Berman JD. Am. J. Trop. Med. Hyg., 1992; 46:296.

62. Pavli A, Maltezou HC. Int. J. Infect. Dis., 2010; 14:e1032.

63. Benhamou E, albou A, destaing F, pugliese J. Bulletin et Memoires de la Societe Medicale des Hopitaux de Paris 1949; 25:1091-1098.

64. Sundar S, Chakravarty J, Agarwal D, Rai M, Murray HW. N. Engl. J. Med., 2010; 362:504-512.

65. Sundar S, Singh A, Rai M, Prajapati VK, Singh AK, Ostyn B. Clin. Infect. Dis., 2012; 55:543-550.

66. Sundar S, Sinha PK, Rai M, Verma DK, Nawin K, Alam S. Lancet 2011; 377:477-486.

67. Chakravarty J, Sundar S. J. Glob. Infect. Dis., 2010; 2:167.

68. Paila YD, Saha B, Chattopadhyay A. Biochem. Biophys. Res. Commun., 2010; 399:429-433.

69. Brown M, Noursadeghi M, Boyle J, Davidson R. Br. J. Dermatol., 2005; 153:203-205.

70. Sindermann H, Croft S, Engel K, Bommer W, Eibl H, Unger C. Med. Microbiol. Immun., 2004; 193; 173-180.

71. Wiwanitkit V. Ther. Clin. Risk Manag., 2012; 8: 323.
72. Costa CHN, Peters NC, Maruyama SR, de Brito EC. PLoS Negl. Trop. Dis., 2011; 5:e943.

73. Spitzer N, Jardim A, Lippert D, Olafson R. Vaccine. 1999; 17:1298-1300.

74. Fernandes AP, Costa MMS, Coelho EAF, Michalick MSM, de Freitas E. Vaccine. 2008; 26:5888-5895.

75. Brodskyn C, De Oliveira CI, Barral A, Barral-Netto M. Exp. Rev. Vaccines., 2003; 2: 705-717.

76. Maltezou HC. BioMed. Res. Int., 2009; 10.

77. Ouellette M, Papadopoulou B. Parasitol. Today 1993; 9; 150-153.

78. Moafi M, Rezvan H, Sherkat R, Taleban R. Int. J. Prev. Med., 2019; 10:95.

79. Sundar S. Tropical Med. Int. Health., 2015; 6:849-854.

80. Mueller M, Ritmeijer K, Balasegaram M, Koummuki Y, Santana MR, Davidson R. Trans R. Soc. Trop. Med. Hyg., 2007; 101(1):19-24.

81. Asilian A, Sharif A, Faghihi G, Enshaeieh S, Shariati F, Siadat AH. Int. J. Dermatol., 2004; 43:736-738.

82. Valencia BM, Miller D, Witzig RS, Boggild AK, Llanos-Cuentas A. PLoS Neg. Trop. Dis., 2013; 7:e2196.

83. Sadeghian G, Nilfroushzadeh MA, Iraji F. Clin. Exp. Dermatol., 2007; 32:371-374.

84. Aronson NE, Wortmann GW, Byrne WR, Howard RS, Bernstein WB, Marovich MA. PLoS Negl. Trop. Dis., 2010; 4:e628.

85. Shamsi SM, Zandi S, Aghaie H, Heshmatkhah A. J. Eur. Acad. Dermatol. Venereol., 2011; 25:587-591.

86. Bassiouny A, El Meshad M, Talaat M, Kutty K, Metawaa B. Br. J. Dermatol., 1992; 107:467-474.

87. Negera E, Gadisa E, Hussein J, Engers H, Kuru T, Gedamu L, Aseffa A. Trans. R. Soc. Trop. Med. Hyg., 2012; 106(8):496-503.

88. Chakravarty J, Sundar S. Expert Opin. Pharmacother., 2019; 20(10):1251-1265.

89. Giladi M, Porta Y, Blatt A, Shmueli E, Wasserman Y, Kirson ED. Antimicrob. Agents Chemother., 2010; 54(8):3212-3218.

90. Wolcott LE, Wheeler PC, Hardwicke HM, Rowley BA. South Med. J., 1969; 62(7):795-801.

91. Hejazi H, Eslami G, Dalimi A. Ann. Trop. Med. Parasito., 2004; 98(1):37-42.

92. Dorsey BM, Cass CL, Cedeño DL, Vallejo R, Jones MA. Pathogens. 2018; 7(4):77.

93. Lopez-Jaramillo P, Ruano C, Rivera J, Terán E, Salazar-Irigoyen R, Esplugues JV, Moncada S. Lancet. 1998; 351:1176-1177.

94. Lopez-Jaramillo P, Rincon MY, Garcia RG, Silva SY, Smith E, Kampeerapappun P. Am. J. Trop. Med. Hyg., 2010; 83:97-10. 
95. Organización Panamericana de la Salud (OPS). Leishmaniasis em las Américas. Recomendaciones para el tratamiento. OPS, Washington, 2013;1-60.

96. Bashir U, Tahir M, Anwar MI, Manzoor F. Pak. J. Med. Sci., 2019; 35(6):1669-1673.

97. Arboleda M, Barrantes S, Úsuga LY, Robledo SM. Rev. Soc. Bras. Med. Trop., 2019; 16(52): e20180211.

98. Ramalho DB, Silva RED, Senna MCR, Moreira HAS, PedrasMJ, Avelar DM. Mem. Inst. Oswaldo. Cruz., 2018; 113(9):e180200.

99. Van Griensven J, Diro E. Infect. Dis. Clin. N. Am., 2019; 33(1):79-99.

100. Monge-Maillo B, López-Vélez R. Drugs. 2013; 17:1863-1888.

101. Sundar S, Rai M, Chakravarty J, Agarwal D, Agrawal N, Vaillant M. Clin. Infect. Dis., 47:1000-1006.

102. Melaku Y, Collin SM, Keus K, Gatluak F, Ritmeijer K, Davidson RN. Am. J. Trop. Med. Hyg., 2007; 77:89-94.

103. Mahajan R, Das P, Isaakidis P, Sunyoto T, Sagili KD, Lima MA. Clin. Infect. Dis., 2015; 61(8):1255-1262.

104. Roatt BM, Cardoso JM, De Brito RCF, Coura-Vital W, Aguiar-Soares RD, Reis AB. Appl. Microbiol. Biotechnol., 2020; 104(19):8105-8116.

105. Ashburn TT, Thor KB. Nat. Rev. Drug Discov., 2004; 3(8):673-683.

106. Pushpakom S, Iorio F, Eyers PA, Escott KJ, Hopper S, Wells A. Nat. Rev. Drug Discov., 2019; 18(1):4158 .

107. Bustamante C, Ochoa R, Asela C, Muskus C. J. Comput. Aided Mol. Des., 2019; 33(9):845-854.

108. Keiser MJ, Setola V, Irwin JJ. Nature. 2009; 462: $175-181$.

109. Tunes LG, Morato RE, Garcia A, Schmitz V, Steindel M. ACS Infect. Dis., 2008; (5):1121-1139.

110. Braga SS. Eur. J. Med. Chem., 2019; 1(183):111660.

111. Le Pape P, Zidane M, Abdala H, More MT. Cell. Biol. Int., 2000; 24:51-56.

112. Antony JP, Fyfe L, Smith H. Trends Parasitol., 2005; $21: 462-468$.

113. Bawa R, Melethil S, Simmons WJ, Harris D. Sci. Tech. Lawyer. 2008; 5: 10-15.

114. Bobo D, Robinson KJ, Islam J, Thurecht KJ, Corrie SR. Pharm. Res., 2016; 33:2373-2387.

115. Moen MD, Lyseng-Williamson KA, Scott LJ. Drugs. 2009; 69:361-392.
116. Li L, Mu Q, Zhang B, Yan B. Analyst. 2010; 135:1519-1530.

117. Saptarshi SR, Duschl A, Lopata AL. J. Nanobiotechnol., 2013; 11:26.

118. Diwan M, Tafaghodi M, Samuel J. J. Control. Release. 2002; 85: 247-262.

119. Shang L, Nienhaus K, Nienhaus GU. J. Nanobiotechnol., 2014; 12:5.

120. Sousa-Batista A, Rossi-Bergmann B. Leishmaniases Reemerging Dis., 2018; 181.

121. Gustafson HH, Holt-Casper D, Grainger DW, Ghandehari H. Nano. Today. 2015; 10:487-510.

122. Costa LE, Alves PT, Carneiro AP, Dias ACS, Fujimura PT, Araujo GR. Int. J. Mol. Sci., 2019; 20: 1812.

123. Meheus F, Balasegaram M, Olliaro P, Sundar S, Rijal S, Faiz MA. PLoS Negl. Trop. Dis., 2010; 4(9):e818.

124. Asthana S, Jaiswal AK, Gupta PK, Pawar VK, Dube A, Chourasia MK. Antimicrob. Agents Chemother., 2013; 57:1714-1722.

125. Do Nascimento TG, Da Silva PF, Azevedo LF, Da Rocha LG, de Moraes Porto ICC, Moura TAL. Nanoscale Res. Lett., 2016; 11:301.

126. Shetty Y, Prabhu P, Prabhakar B. Int. J. Pharm., 2019; 558:29-42.

127. Peer D, Karp JM, Hong S, Farokhzad OC, Margalit R, Langer R. Nat. Nanotechnol., 2007; 2:751-760.

128. Souto DE, Fonseca AM, Barragan JT, de CS Luz R, Andrade HM, Damos FS. Biosens. Bioelectron., 2015; 70:275-281.

129. Doroud D, Zahedifard F, Vatanara A, Najafabadi AR, Taslimi Y, Vahabpour R. J. Control. Release. 2011; 153:154-162.

130. Sekhon BS, Saluja V. Int. J. Pharm. Front. Res., 2011; 1(1): 101-109.

131. Danesh-Bahreini MA, Shokri J, Samiei A, KamaliSarvestani E, Barzegar-Jalali M, Mohammadi-Samani S. Int. J. Nanomedicine., 2011; 6:835.

132. Athanasiou E, Agallou M, Tastsoglou S, Kammona O, Hatzigeorgiou A, Kiparissides C, Karagouni E. Front. Immunol., 2017; 8:684.

133. Agallou M, Margaroni M, Athanasiou E, Toubanaki DK, Kontonikola K, Karidi K, Kammona O, Kiparissides C, Karagouni E. PLoS Negl. Trop. Dis., 2017; 11(1):e0005311.

134. Urena-Búrquez LA, García-Rendon A, Rochín-Wong S, Tejeda-Mansir A. Fuel. 2019; 235:1077-1082. 